\title{
LA SOSTENIBILIDAD AMBIENTAL REGIONAL: UNA PROPUESTA METODOLÓGICA PARA SU ESTUDIO MÁS ALLÁ DE LA ECOLOGÍA URBANA
}

\author{
MARÍA VICTORIA PINZÓN BOTERO ${ }^{1}$ \\ ISABEL CRISTINA ECHEVERRI ÁLVAREZ ${ }^{2}$ \\ mvpinzonb@unal.edu.co, fisin.2011@gmail.com
}

Manizales, 2011-09-15 (Rev. 2012-02-25)

\section{RESUMEN}

El sistema territorial a escala regional posee una mayor capacidad para permitir la disipación y asimilación de los diferentes impactos ambientales producidos en el sistema urbano. Los espacios urbanos considerados como los principales promotores de graves impactos ambientales, deben ser analizados como sistemas interdependientes de otros territorios, estableciéndose así, la conformación de sistemas regionales de gran importancia para el análisis de la capacidad de sostenibilidad ambiental. El método de estudio de dicha capacidad se convierte en el objetivo del presente artículo (fase cuatro de la metodología), cuyo producto es uno de los resultados de la investigación realizada en el marco de la tesis de maestría de una de las autoras. La selección de 22 variables ambientales estratégicas producto del análisis de dos grupos de variables ambientales estudiadas por la investigación, se convierten en las condicionantes principales que determinarán con mayor o menor éxito, la sostenibilidad ambiental de un territorio configurado como región.

\section{PALABRAS CLAVE}

Capacidad ambiental, ciudad-región, metodologías, sostenibilidad ambiental.

REGIONAL ENVIRONMENTAL SUSTAINABILITY: A METHODOLOGICAL PROPOSAL FOR A STUDY BEYOND UBRBAN ECOLOGY

\begin{abstract}
The territorial system at a regional scale has a greater capacity to allow dissipation and assimilation of different environmental impacts produced in the urban system. Urban spaces, considered as the main promoters of serious environmental impacts, should be analyzed as interdependent systems of other territories, establishing this way the conformation of regional systems of great importance for analyzing the environmental sustainability capacity. The method of study of such capacity is the aim of this article (phase four of the methodology), which product is one of the results of the research conducted within the framework of the Master's Degree thesis requirement of one of the authors. The selection of Twenty-two strategic environmental variables resulting from the analysis of two groups of environmental variables studied within this research become the main determinants that will determine the degree of success of environmental sustainability of an area set up as a region.
\end{abstract}

\section{KEY WORDS}


Environmental capacity, city-region, methodologies, environmental sustainability.

\section{INTRODUCCIÓN}

Actualmente existe una creciente preocupación mundial por disminuir significativamente los impactos ambientales negativos que ponen en grave riesgo la vida sobre el planeta. Diferentes debates locales, nacionales e internacionales, se han gestado con respecto al desarrollo sostenible en diferentes escalas, con predominio de propuestas que giran en torno a la comprensión de las relaciones que operan al interior de una ciudad, y de ésta con su entorno inmediato, como un sistema al cual se asocian las mayores responsabilidades en materia de servicios y de afectación sobre la capacidad ambiental del territorio. Al respecto Odum (como se cita en Zuluaga, 2003) define la ciudad:

"como un sistema dependiente de amplias zonas limítrofes y no limítrofes, para la energía, los alimentos, las fibras, el agua y otros materiales para el sostenimiento de la vida misma y para el uso comercial e industrial".

En el mismo sentido Naredo (1997), plantea que

"es necesario relacionar el tamaño de las ciudades y sus exigencias en recursos y el modo de abastecerlas, con las posibilidades que le brinda el territorio local y global de referencia".

Visualizándose la necesidad de incorporar estrategias de sostenibilidad ambiental que consideren dichas relaciones, debido a que:

"cuando de esta manera se tome conciencia de la interacción que existe entre la ciudad y la región, entre los aspectos rurales y urbanos del ambiente, sea tanto a pequeña como a gran escala, un sentido nuevo de la forma encontrará espacio en la arquitectura de la ciudad" (Bettini, 1998).

Así mismo, Yunén (1997), afirma que:

"el estudio del ambiente de una ciudad debe partir, entonces, del contexto en que ésta se desenvuelve, para así determinar la escala (hogar, barrio, sector, ciudad, municipio, cuenca, región, país, continente) donde con mayor frecuencia e intensidad se presentan o se producen problemas ambientales".

La importancia de concebir los espacios urbanos conectados y dependientes de su contexto cercano e inclusive en muchos casos más lejano, se convierte en un aspecto imprescindible para la comprensión y análisis de las dinámicas territoriales y ambientales de estas áreas pobladas. Por tanto, estos territorios deben ser abordados desde concepciones sistémicas que impidan separar los aspectos ambientales significativos de otros elementos no tan visibles desde el discurso ambiental (Ángel, 1996). De esta manera Bertalanffy (citado por Leal, 2006) concibe el sistema como "un conjunto de elementos en interacción”, y en sentido más amplio, como la agrupación de elementos 
interconectados que establecen interdependencias entre sí, y de las cuales emergen propiedades que tienden a hacer más complejo el sistema. Bajo este enfoque (Morin, 2000), dice que es necesario reconocer y estudiar la ciudad como un sistema abierto en estrecha relación con un territorio externo donde predomina el patrimonio natural, asociado principalmente con el medio de soporte de las actividades urbanitas de donde se obtienen recursos y energía para el sostenimiento de la ciudad, así como, también con el medio que permite la asimilación de los residuos que se generan en su interior, emergiendo sistemas como el municipal y el regional, con territorios más extensos, diversos y, por tanto, más complejos, donde es más factible el trabajo en pro de la sostenibilidad ambiental.

La región como unidad geográfica de interés para entender el sistema territorial ambiental, se concibe como el área delimitada con base en criterios territoriales y de planificación ambiental, integrada por un conjunto de ciudades y su medio rural, conformada por núcleos poblacionales jerarquizados entre los cuales se generan relaciones similares favorecidas por la diversidad de la oferta natural y sociocultural, las cuales le confieren cierta especificidad que la distingue dentro de un contexto mayor (Fals, 1996).

Al respecto la CEPAL, plantea que:

"el reto será evitar la fragmentación de los territorios nacionales y regionales, en donde las ciudades deberán alternativamente mirar al mundo y a sí mismas con sus regiones, al futuro y al pasado, garantizando un manejo integrado y coherente del territorio" (p. 56).

La sostenibilidad definida por Parra (1997), como:

"la viabilidad de una actividad (o de un conjunto de actividades, de un proceso, de toda una sociedad, etc.) en el tiempo, esto es, su posibilidad de supervivencia en el futuro sin comprometerlo o situarlo en condiciones precarias".

Fusionándose al concepto de sistema regional, para entender la sostenibilidad ambiental regional. En este sentido la sostenibilidad ambiental regional, se define como la permanencia en el tiempo de las capacidades biofísica, socio-espacial, económica y político institucional de un territorio para albergar una población de manera confortable, organizada en núcleos poblacionales, y entornos rurales diversos, con potencialidades y restricciones ambientales específicas, en función de las complejas interacciones que emergen entre hombre y naturaleza que, interrelacionadas con las diferentes fuerzas ejercidas sobre él, permiten que éste persista y sea apto para el desarrollo de diversas funciones y de su viabilidad en el tiempo. Según Naredo, (1997):

"la calidad de vida e incluso la supervivencia de la humanidad estará en buena parte condicionada por su capacidad para conocer y controlar la relación de las ciudades con su medio ambiente".

En el camino hacia el estudio de la sostenibilidad ambiental, importantes agencias internacionales, gobiernos y autores especializados, han propuesto y aplicado diferentes metodologías, entendidas como estructuras lógicas que de forma ordenada, clara, precisa, detallada, y con rigor científico, presentan una forma adecuada de trabajar por este 
objetivo. Dichas propuestas realizan aportes conceptuales principalmente al tema de la sostenibilidad ambiental, pero pocas se enfocan en la sostenibilidad ambiental regional. Igualmente, se caracterizan por el uso de variables, como un elemento ordenador donde de manera jerárquica se agrupa la información, y cuyo comportamiento, incide en la sostenibilidad o insostenibilidad de un sistema (Rayén, 2001).

Se establece, por ende, en esta investigación, que las variables ambientales estratégicas corresponden a aquellas variables esenciales, cuyo comportamiento incide de manera directa y significativa sobre los distintos tipos de capacidad de sostenibilidad ambiental regional. Se entenderá el concepto de capacidad como la disminución o aumento de los tipos de capital asociados que, por tanto, condicionan la sostenibilidad del sistema territorial regional.

Se tuvo en cuenta también en esta investigación, la dinámica territorial de una región, entendida como la transformación, bien sea material o intangible, que experimenta este sistema en función de las potencialidades y restricciones ambientales asociadas al territorio, y como respuesta a los diversos modos de influencia y presión que ejercen sobre éste los actores sociales. Fue seleccionado como caso referente de la investigación la región suroriental del departamento del Valle del Cauca, aplicando este ejercicio, para identificar variables importantes a considerar como elemento adicional a los modelos estudiados.

La propuesta de un diseño metodológico a partir de otros modelos estudiados, es parte fundamental de esta investigación, donde su objetivo fue la selección de variables ambientales estratégicas, que permitan posteriormente, a través de sus respuestas (indicadores) y análisis, reconocer la capacidad de sostenibilidad ambiental de una región. Dicha propuesta se constituye en el resultado de la revisión de las variables contenidas en diferentes proposiciones y/o modelos sobre sostenibilidad ambiental, y en la identificación de los principales componentes del área de trabajo, estudiados bajo las dinámicas territoriales y las potencialidades, y restricciones ambientales de la región tomada como caso referente para esta investigación.

\section{MATERIALES Y MÉTODOS}

El presente artículo muestra el desarrollo de la fase 4 del trabajo de investigación correspondiente al diseño metodológico, principalmente a través de herramientas cualitativas. El trabajo de investigación completo se estructuró en cuatro fases: la primera fase, corresponde a la elaboración de un referente teórico-conceptual compuesto por tres componentes: (1) relacionado con los términos y conceptos básicos que se asocian directa e indirectamente con la sostenibilidad ambiental, a través de los cuales, se realizó una primera introducción al tema (sistemas y complejidad, desarrollo sostenible y/o sustentable, capacidad de sostenibilidad ambiental, metodología para el estudio de la sostenibilidad ambiental, planificación ambiental y ordenamiento territorial), (2) el concepto de región como modelo territorial objeto de estudio y (3) la sostenibilidad ambiental regional.

En la segunda fase, se revisaron distintas metodologías propuestas por varios autores a nivel internacional y nacional (ver Tabla 1), las cuales fueron clasificadas en: metodologías con énfasis en indicadores tipo índice, en indicadores de sostenibilidad y de desarrollo sostenible 
(Velásquez, 2005), indicadores que estudian la sostenibilidad ecológica urbana, y que permiten planificar y ordenar cuencas hidrográficas, y finalmente, las que integran indicadores de potencial frente a los Compromisos de Aalborg particularmente aplicables a ciudades europeas (Antequera, 2004). Las variables contenidas en cada uno de los modelos fueron reagrupadas para ser estudiadas por dimensión teniendo en cuenta la metodología de estudio territorial por subsistemas.

Tabla 1. Modelos revisados y dimensiones de reagrupación de variables para su estudio.

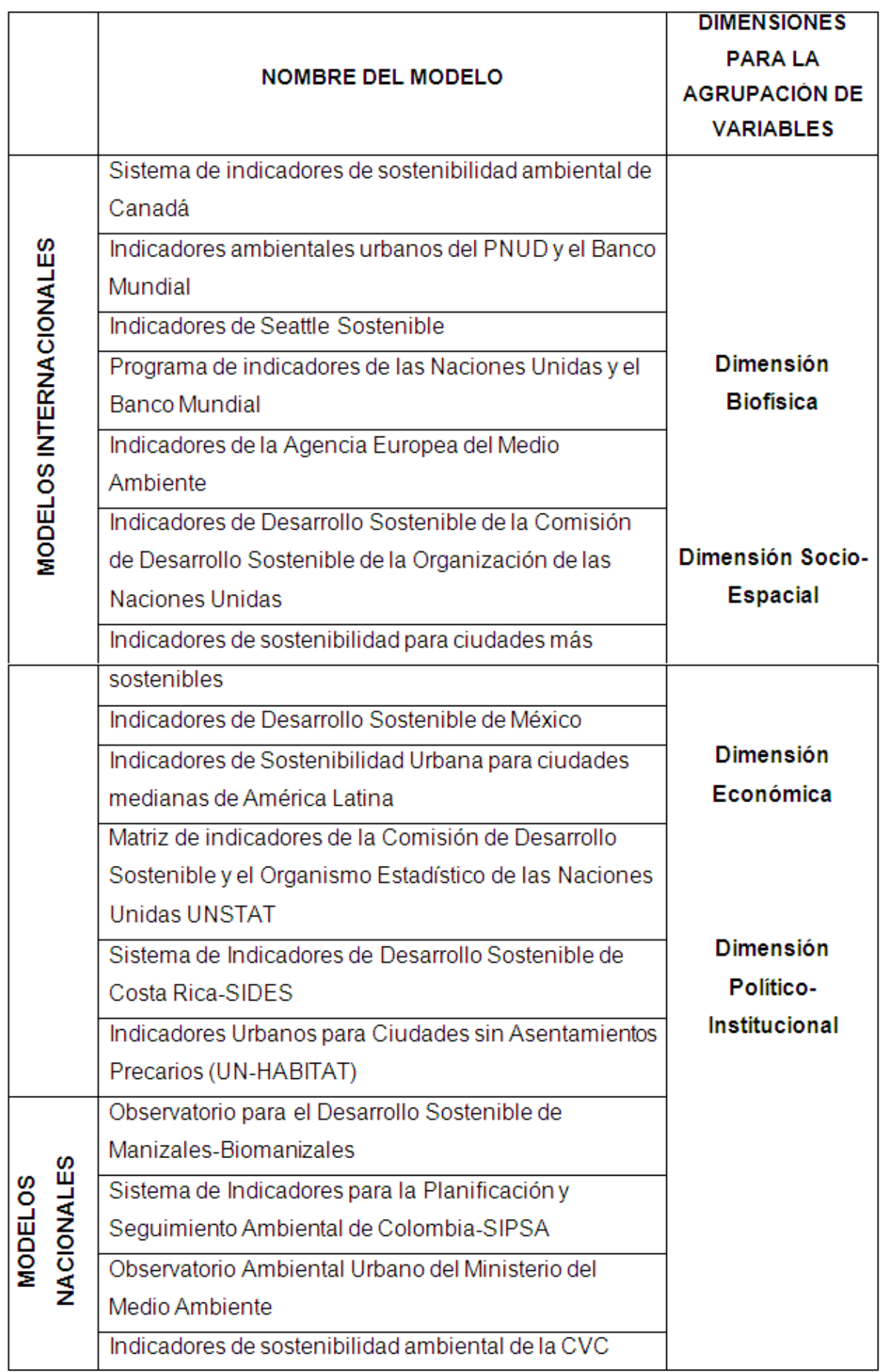


Las fases 1 y 2, fueron complementadas con la fase 3 de la metodología, al abordar la observación de la región como unidad geográfica, y como área de estudio referente de la investigación. La región suroriental del departamento del Valle del Cauca, fue escogida para este fin, identificando en ella sus principales dinámicas territoriales, con el fin de encontrar un conjunto de potencialidades y restricciones ambientales importantes a esta escala que nos permitieran corroborar la relevancia de las variables ambientales identificadas en los modelos revisados, y complementar otras variables no previstas en ellos, que por su impacto o trascendencia en la escala regional, debían hacer parte de la metodología planteada.

La fase 4 hace referencia a la construcción del diseño metodológico para estudiar la capacidad de sostenibilidad ambiental regional, para lo cual se llevaron a cabo tres momentos:

Primer momento: se constituye en la identificación de las Variables Ambientales asociadas a la Sostenibilidad Ambiental Regional (VASAR), teniendo en cuenta los resultados de las anteriores fases metodológicas y comparando las variables ambientales contenidas en cada una de las diferentes propuestas estudiadas para observar similitud y coincidencia entre ellas, y así obtener un primer grupo de variables ambientales.

Al respecto se tuvieron en cuenta las siguientes consideraciones:

I) Una variable ambiental que define la capacidad biofísica y socio-espacial, es representativa si está contenida por lo menos en 3 propuestas y/o modelos revisados.

II) Una variable ambiental que define la capacidad económica y político institucional, es representativa si está contenida por lo menos en 2 propuestas y/o modelos revisados.

La anterior diferenciación en la consideración, responde al énfasis que en décadas anteriores, se le ha dado a variables asociadas a los componentes biofísico y socio-espacial, frente a la poca atención con la que contaron las variables asociadas a los componentes económico y político institucional, reconocidos en los últimos años como factores determinantes e influyentes en el comportamiento ambiental, según lo estudiado en modelos como el sistema de indicadores de sostenibilidad ambiental de Canadá, los indicadores de la Agencia Europea del Medio Ambiente, la matriz de indicadores de la Comisión de Desarrollo Sostenible y el Organismo Estadístico de las Naciones Unidas UNSTAT, entre otros.

Posteriormente a este grupo de variables seleccionadas, se aplican seis criterios establecidos a partir de la definición de los principios de la Sostenibilidad Ambiental Regional, configurados como resultado del análisis de la información teórico-conceptual y metodológica recopilada. Igualmente, se verifica el peso de las variables en el modelo a proponer, al tener en cuenta la realidad regional del estudio de caso referenciado (región suroriental del departamento del Valle del Cauca). Para la definición de las VASAR, se tuvo en cuenta que los puntajes fueran iguales o mayores al valor de la suma resultante entre el promedio aritmético del mayor y el menor valor de las calificaciones obtenidas para cada variable ambiental evaluada.

Los seis criterios definidos según los principios de la Sostenibilidad Ambiental Regional a los cuales, se sometió el listado de variables ambientales fueron los siguientes: 
Principio 1: la sostenibilidad ambiental regional estudia una región como un sistema abierto con unidades territoriales que se interrelacionan entre sí, de acuerdo al nivel de influencia sobre el territorio (Tabla 2). Para este caso, y para los principios 2 y 4 , solo se evalúa con los parámetros 0 y 3 , debido a que los niveles de evaluación para los criterios correspondientes son específicos para condicionantes de alta y baja magnitud.

Tabla 2. Parámetros para la evaluación del Nivel de Influencia.

\begin{tabular}{|c|c|c|}
\hline CRITERIO & DEFINICIÓN & FORMA DE APLICACION \\
\hline $\begin{array}{l}\text { Nivel de } \\
\text { influencia sobre } \\
\text { el territorio } \\
\text { (NIT) }\end{array}$ & $\begin{array}{l}\text { Hace referencia al poder } \\
\text { que la variable ambiental } \\
\text { ejerce sobre un territorio, } \\
\text { teniendo en cuenta que } \\
\text { ésta puede influenciar a } \\
\text { unidades territoriales de } \\
\text { forma específica o a } \\
\text { todas las unidades } \\
\text { territoriales y por lo tanto } \\
\text { a la región en su } \\
\text { conjunto. }\end{array}$ & $\begin{array}{l}\text { Se evalúa con base en los siguientes } \\
\text { parámetros: } \\
0=\text { si la influencia de la variable } \\
\text { ambiental es local, es decir sobre cada } \\
\text { unidad territorial de forma } \\
\text { independiente. } \\
3=\text { si la influencia de la variable } \\
\text { ambiental es general, es decir sobre } \\
\text { toda la región. } \\
\text { Los valores a asignar se introducen en } \\
\text { la matriz de selección. }\end{array}$ \\
\hline
\end{tabular}

Principio 2: la sostenibilidad ambiental regional, garantiza el mínimo consumo de capital no renovable (energía), para alimentar los otros tipos de capital (Tabla 3).

Tabla 3. Parámetros para evaluar el consumo de energía no renovable (CENR).

\begin{tabular}{|c|c|c|}
\hline CRITERIO & DEFINICIÓN & FORMA DE APLICACIÓN \\
\hline $\begin{array}{ll}\text { Consumo } & \text { de } \\
\text { energía } & \text { no } \\
\text { renovable } & \\
\text { (CENR) } & \end{array}$ & $\begin{array}{l}\text { Se refiere a las } \\
\text { necesidades de consumo } \\
\text { de combustibles fósiles } \\
\text { de la variable ambiental, } \\
\text { como una forma de } \\
\text { energía no renovable } \\
\text { altamente contaminante. }\end{array}$ & $\begin{array}{l}\text { Se evalúa con base en los siguientes } \\
\text { parámetros: } \\
0 \text { = algún tipo de consumo de energía } \\
\text { no renovable por parte de la variable } \\
\text { ambiental. } \\
3 \text { = ningún consumo de energía no } \\
\text { renovable por parte de la variable } \\
\text { ambiental. } \\
\text { Los valores a asignar se introducen en } \\
\text { la matriz de selección. }\end{array}$ \\
\hline
\end{tabular}

Principio 3: la sostenibilidad ambiental regional procura diversificar el sistema, y hacerlo dependiente de varios tipos de recursos naturales renovables (Tabla 4). 
Tabla 4. Parámetros para la evaluación de la Dependencia de Recursos Naturales Renovables (DRNR).

\begin{tabular}{|c|c|c|}
\hline CRITERIO & DEFINICIÓN & FORMA DE APLICACIÓN \\
\hline $\begin{array}{l}\text { Dependencia } \\
\text { de recursos } \\
\text { naturales } \\
\text { renovables } \\
\text { (DRNR) }\end{array}$ & $\begin{array}{l}\text { Se refiere al grado de } \\
\text { dependencia directa de la } \\
\text { variable ambiental sobre } \\
\text { los recursos naturales } \\
\text { renovables en función del } \\
\text { número de recursos } \\
\text { naturales renovables } \\
\text { implicados. }\end{array}$ & 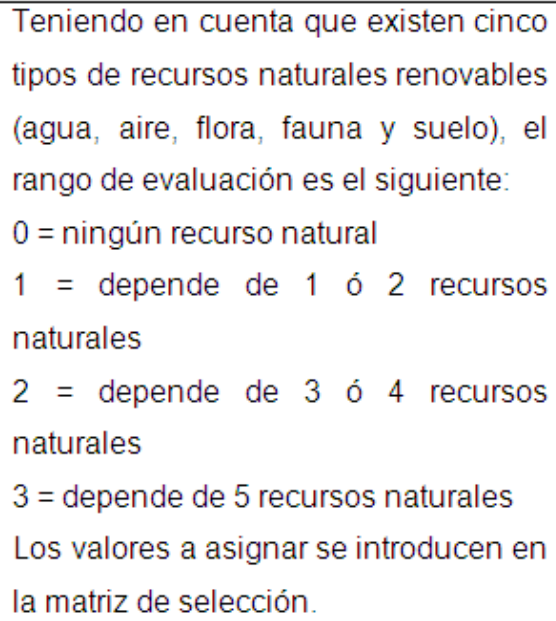 \\
\hline
\end{tabular}

Principio 4: la sostenibilidad ambiental regional considera las ventajas competitivas del sistema regional en su conjunto (Tabla 5).

Tabla 5. Parámetros para evaluar la Contribución de la Variable Ambiental a las ventajas competitivas de la región (CVC).

\begin{tabular}{|c|c|c|}
\hline CRITERIO & DEFINICIÓN & FORMA DE APLICACIÓN \\
\hline $\begin{array}{l}\text { Contribución de } \\
\text { la variable } \\
\text { ambiental a las } \\
\text { ventajas } \\
\text { competitivas de } \\
\text { la región (CVC) }\end{array}$ & $\begin{array}{l}\text { Hace referencia a la } \\
\text { manera cómo la variable } \\
\text { ambiental influencia } \\
\text { positiva o negativamente } \\
\text { el desarrollo de ventajas } \\
\text { competitivas para la } \\
\text { región en su conjunto, de } \\
\text { tal forma que permita } \\
\text { visualizar aquellas que } \\
\text { contribuyen a otorgarle } \\
\text { un mayor potencial. }\end{array}$ & $\begin{array}{l}\text { Se evalúa con base en los siguientes } \\
\text { parámetros: } \\
0=\text { si no contribuye a las ventajas } \\
\text { competitivas de la región. } \\
3=\text { si contribuye a las ventajas } \\
\text { competitivas de la región. } \\
\text { Los valores a asignar se introducen en } \\
\text { la matriz de selección. }\end{array}$ \\
\hline
\end{tabular}

Principio 5: la sostenibilidad ambiental regional tiende a disminuir la degradación ambiental debido a la mayor capacidad de disipación de la región en su conjunto, la cual se ve representada en el nivel de entropía generada (Tabla 6). 
Tabla 6. Parámetros para la evaluación del Nivel de Entropía Generada (NEG).

\begin{tabular}{|c|c|c|}
\hline CRITERIO & DEFINICIÓN & FORMA DE APLICACIÓN \\
\hline $\begin{array}{l}\text { Nivel de } \\
\text { entropia } \\
\text { generada } \\
\text { (NEG) }\end{array}$ & $\begin{array}{l}\text { Hace referencia al } \\
\text { desorden sobre el medio } \\
\text { externo que la variable } \\
\text { ambiental es factible de } \\
\text { generar para mantener su } \\
\text { propio orden interno. Este } \\
\text { desorden está } \\
\text { representado en la } \\
\text { generación de residuos } \\
\text { sólidos, aguas residuales } \\
\text { y emisiones al aire de } \\
\text { cualquier tipo (gases, } \\
\text { partículas). } \\
\text { El desorden se puede } \\
\text { caracterizar en función } \\
\text { del número de tipos de } \\
\text { entropía generada. }\end{array}$ & $\begin{array}{l}\text { Se evalúa con base en los siguientes } \\
\text { parámetros: } \\
1 \text { = si la variable ambiental genera los } \\
3 \text { tipos de entropía descritos. } \\
2 \text { = si la variable ambiental genera } 1 \text { ó } \\
2 \text { tipos de entropía descritos. } \\
3 \text { = si la variable ambiental no genera } \\
\text { ningún tipo de entropía. } \\
\text { Los valores a asignar se introducen en } \\
\text { la matriz de selección. }\end{array}$ \\
\hline
\end{tabular}

Principio 6: la sostenibilidad ambiental regional integra los distintos tipos de capacidad biofísica, socio-espacial, económica y político institucional (Tabla 7).

Tabla 7. Parámetros para la evaluación de la Influencia sobre Tipos de Capacidad (ITC).

\begin{tabular}{|c|c|c|}
\hline CRITERIO & DEFINICIÓN & FORMA DE APLICACIÓN \\
\hline $\begin{array}{l}\text { Influencia } \\
\text { directa de la } \\
\text { variable sobre } \\
\text { los distintos } \\
\text { tipos de } \\
\text { capacidad } \\
\text { (ITC) }\end{array}$ & $\begin{array}{l}\text { Corresponde al carácter } \\
\text { estratégico de una } \\
\text { variable ambiental de } \\
\text { acuerdo con su influencia } \\
\text { sobre los distintos tipos } \\
\text { de capacidad: biofísica, } \\
\text { socio-espacial, } \\
\text { económica o político } \\
\text { institucional. }\end{array}$ & $\begin{array}{l}\text { Se evalúa con base en los siguientes } \\
\quad \text { parámetros: } \\
0=\text { si no influencia ningún tipo de } \\
\text { capacidad. } \\
1=\text { si influencia } 1 \text { tipo de capacidad. } \\
2=\text { si influencia } 2 \text { tipos de capacidad. } \\
3=\text { si influencia } 3 \text { ó } 4 \text { tipos de } \\
\text { capacidad. } \\
\text { Los valores a asignar se introducen en } \\
\quad \text { la matriz de selección. }\end{array}$ \\
\hline
\end{tabular}

Segundo momento: la identificación de las Variables Ambientales asociadas a las Dinámicas Territoriales (VADIT), bajo el conocimiento de los temas estratégicos enunciados por cada dinámica territorial, producto de la reflexión y el análisis de cada una de las potencialidades 
y restricciones ambientales territoriales del referente de investigación (región suroriental del departamento del Valle del Cauca).

Tercer momento: definición de las Variables Ambientales Estratégicas para el estudio de la Sostenibilidad Ambiental Regional (VAESAR), a partir de un procedimiento de reflexión alrededor de las propuestas VASAR y VADIT, para la identificación de variables ambientales comunes entre ellas, que respondieran al concepto VAESAR y de otras, como las variables energía, infraestructura de soporte y flujos de bienes y servicios que aunque no resultaron comunes, se concibieron de especial importancia, principalmente por el consumo de recursos naturales (capital natural), o por su poder para asimilar la entropía generada por el sistema.

\section{RESULTADOS Y DISCUSIÓN}

En este artículo solo se presentan los resultados de la fase 4 de la metodología descrita, que corresponden al diseño metodológico propuesto para la definición de las variables ambientales estratégicas en el estudio de la capacidad de sostenibilidad ambiental regional. Se apoya la discusión final de las variables ambientales estratégicas con el documento "Los objetivos de desarrollo del milenio en América Latina y el Caribe: retos, acciones y compromisos" de 2004, del Banco Interamericano de Desarrollo, al ser este un lineamiento fundamental para el desarrollo ambiental en Colombia y América Latina.

El primer momento produce como resultado la identificación de las variables ambientales asociadas a la sostenibilidad ambiental regional (VASAR), conformadas por un listado general de variables ambientales observadas y analizadas en las distintas propuestas y/o modelos revisados. Los resultados se observan en la tabla 8 : 
Tabla 8. Listado de variables ambientales consolidadas de acuerdo a la coincidencia entre propuestas y/o modelos revisados.

\begin{tabular}{|c|c|c|c|}
\hline $\begin{array}{l}\text { Dimensión } \\
\text { Biofisica }\end{array}$ & $\begin{array}{c}\text { Dimensión Socio- } \\
\text { espacial }\end{array}$ & $\begin{array}{l}\text { Dimensión } \\
\text { Económica }\end{array}$ & $\begin{array}{c}\text { Dimensión } \\
\text { Político- } \\
\text { institucional }\end{array}$ \\
\hline Clima & Tránsito y & Empleo & Participación en \\
\hline Biodiversidad & transporte Energía & Remuneración al trabajo & política Inversión \\
\hline Recursos marinos & Población & Ingreso y distribución & institucional \\
\hline Contaminación & Calidad del hábitat & del ingreso & Inversión ambiental \\
\hline atmosférica & Salud & Participación en la & Capacidad \\
\hline Agua & Vivienda & producción nacional & institucional \\
\hline Contaminación & Seguridad & Pobreza urbana & Participación \\
\hline hídrica Bosques & ciudadana & Finanzas & comunitaria \\
\hline Agricultura & Educación & Ejecución presupuestal & Gestión \\
\hline Residuos sólidos & Recreación y & Producción más limpia & gubernamental \\
\hline Suelo/uso del suelo & cultura & Comportamiento fiscal & Gestión no \\
\hline Contaminación & Seguridad social & local & gubernamental \\
\hline sónica & Servicios públicos & Comportamiento & Gestión del sector \\
\hline Riesgos & domiciliarios & económico por sectores & privado \\
\hline $\begin{array}{c}\text { Contaminación } \\
\text { visual }\end{array}$ & & Comercio exterior & Gestión ambiental \\
\hline
\end{tabular}

Los resultados de la aplicación de los seis criterios definidos como los principios de la Sostenibilidad Ambiental Regional, diseñados para la evaluación a través de matrices en las que se han consignado las distintas variables ambientales se observan en la Tabla 9:

Tabla 9. Matrices de selección de Variables Ambientales asociadas a la Sostenibilidad Ambiental Regional VASAR según la aplicación de los principios. 


\begin{tabular}{|c|c|c|c|c|c|c|c|}
\hline \multicolumn{8}{|c|}{ DIMENSIÓN BIOFÍSICA } \\
\hline \multirow{2}{*}{$\begin{array}{l}\text { VARIABLE } \\
\text { AMBIENTAL }\end{array}$} & \multicolumn{7}{|c|}{ CRITERIOS } \\
\hline & NIT & ENR & DRNR & CVC & NEG & ITC & Total \\
\hline Clima & 3 & 3 & 1 & 3 & 3 & 3 & 16 \\
\hline Biodiversidad & 3 & 3 & 2 & 3 & 3 & 2 & 16 \\
\hline Recursos marinos & 3 & 3 & 1 & 3 & 3 & 2 & 15 \\
\hline $\begin{array}{l}\text { Contaminación } \\
\text { atmosférica }\end{array}$ & 3 & 3 & 1 & 0 & 2 & 2 & 11 \\
\hline Agua & 3 & 3 & 0 & 3 & 3 & 3 & 15 \\
\hline Contaminación hídrica & 3 & 3 & 1 & 0 & 2 & 2 & 11 \\
\hline Bosques & 3 & 3 & 2 & 3 & 3 & 2 & 16 \\
\hline Residuos sólidos & 3 & 3 & 0 & 0 & 2 & 2 & 10 \\
\hline Suelo/uso del suelo & 3 & 3 & 2 & 3 & 3 & 3 & 17 \\
\hline Contaminación sónica & 0 & 3 & 0 & 0 & 2 & 1 & 6 \\
\hline Vulnerabilidad y riesgos & 3 & 3 & 1 & 0 & 3 & 3 & 13 \\
\hline Contaminación visual & 0 & 3 & 0 & 0 & 3 & 2 & 8 \\
\hline \multicolumn{8}{|c|}{ DIMENSIÓN SOCIOESPACIAL } \\
\hline VARIABLE & \multicolumn{7}{|c|}{ CRITERIOS } \\
\hline AMBIENTAL & NIT & CENR & DRNR & CVC & NEG & ITC & Total \\
\hline Tránsito y transporte & 3 & 0 & 1 & 3 & 2 & 3 & 12 \\
\hline Población & 3 & 0 & 2 & 3 & 2 & 3 & 13 \\
\hline Energía & 3 & 0 & 1 & 3 & 2 & 3 & 12 \\
\hline Calidad del hábitat & 0 & 3 & 0 & 3 & 3 & 1 & 10 \\
\hline Salud & 3 & 3 & 2 & 3 & 2 & 1 & 14 \\
\hline Vivienda & 3 & 3 & 1 & 3 & 3 & 1 & 14 \\
\hline Seguridad ciudadana & 3 & 3 & 0 & 3 & 3 & 1 & 13 \\
\hline Educación & 3 & 3 & 0 & 3 & 3 & 1 & 13 \\
\hline Recreación y cultura & 3 & 3 & 1 & 3 & 3 & 3 & 16 \\
\hline Seguridad social & 3 & 3 & 0 & 3 & 3 & 1 & 13 \\
\hline Servicios públıcos & 3 & 0 & 1 & 3 & 1 & 3 & 11 \\
\hline \multicolumn{8}{|c|}{ DIMENSIÓN ECONÓMICA } \\
\hline VARIABLE & \multicolumn{7}{|c|}{ CRITERIOS } \\
\hline AMBIENTAL & NIT & CENR & DRNR & CVC & NEG & ITC & Total \\
\hline Agricultura & 3 & 0 & 3 & 3 & 2 & 2 & 13 \\
\hline Empleo & 3 & 3 & 0 & 3 & 3 & 2 & 14 \\
\hline Remuneración al trabajo & 3 & 3 & 0 & 3 & 3 & 2 & 14 \\
\hline $\begin{array}{l}\text { Ingreso y distribución } \\
\text { del ingreso }\end{array}$ & 3 & 3 & 0 & 3 & 3 & 2 & 14 \\
\hline $\begin{array}{l}\text { Participación en la } \\
\text { producción nacional }\end{array}$ & 3 & 0 & 3 & 3 & 1 & 1 & 11 \\
\hline Pobreza urbana & 3 & 3 & 0 & 0 & 3 & 2 & 11 \\
\hline Finanzas & 3 & 3 & 0 & 3 & 3 & 1 & 13 \\
\hline Ejecución presupuestal & 3 & 3 & 0 & 3 & 3 & 2 & 14 \\
\hline Producción más limpia & 3 & 3 & 3 & 3 & 1 & 3 & 16 \\
\hline $\begin{array}{l}\text { Comportamiento fiscal } \\
\text { local }\end{array}$ & 3 & 3 & 0 & 3 & 3 & 2 & 14 \\
\hline $\begin{array}{l}\text { Comportamiento } \\
\text { económico por sectores }\end{array}$ & 3 & 0 & 3 & 3 & 1 & 1 & 11 \\
\hline Comercio exterior & 3 & 0 & 3 & 3 & 1 & 1 & 11 \\
\hline \multicolumn{8}{|c|}{ DIMENSIÓN POLÍTICO INSTITUCIONAL } \\
\hline VARIABLE & \multicolumn{7}{|c|}{ CRITERIOS } \\
\hline AMBIENTAL & NIT & CENR & DRNR & CVC & NEG & ITC & Total \\
\hline Participación en política & 3 & 3 & 0 & 3 & 3 & 2 & 14 \\
\hline Inversión institucional & 3 & 3 & 0 & 3 & 3 & 1 & 13 \\
\hline Inversión ambiental & 3 & 3 & 0 & 3 & 3 & 3 & 15 \\
\hline Capacidad institucional & 3 & 3 & 0 & 3 & 3 & 1 & 13 \\
\hline Participación & 3 & 3 & 0 & 3 & 3 & 2 & 14 \\
\hline ciudadana/comunitaria & & & & & & & \\
\hline $\begin{array}{l}\text { Participación } \\
\text { ciudadana/comunitaria }\end{array}$ & 3 & 3 & 0 & 3 & 3 & 2 & 14 \\
\hline Gestión gubernamental & 3 & 3 & 0 & 3 & 3 & 1 & 13 \\
\hline $\begin{array}{l}\text { Gestión no } \\
\text { gubernamental }\end{array}$ & 3 & 3 & 0 & 3 & 3 & 1 & 13 \\
\hline $\begin{array}{c}\text { Gestión del sector } \\
\text { privado }\end{array}$ & 3 & 3 & 0 & 3 & 3 & 1 & 13 \\
\hline Gestión ambiental & 3 & 3 & 0 & 3 & 3 & 3 & 15 \\
\hline
\end{tabular}


De manera seguida, y definido el puntaje línea base, se seleccionaron las Variables Ambientales asociadas a la Sostenibilidad Ambiental Regional (VASAR). A continuación, como producto del análisis de las dinámicas territoriales de una región como referente de investigación (región suroriental del departamento del Valle del Cauca), se identificaron las variables ambientales asociadas a dichas dinámicas, agrupándolas según las distintas dimensiones ambientales (ver figura. 1).

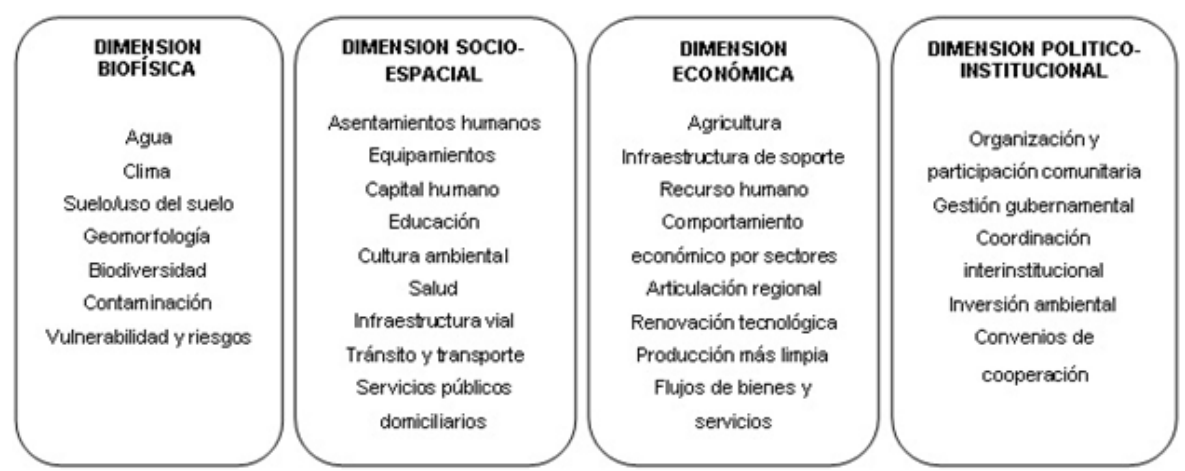

Figura 1. Variables Ambientales asociadas a las Dinámicas Territoriales (VADIT).

Finalmente, se eligieron 22 variables ambientalmente estratégicas, que permiten estudiar la capacidad de sostenibilidad ambiental regional (VAESAR), producto del ejercicio comparativo entre ambas propuestas de variables agrupadas en cada dimensión, donde se observa la similitud entre el contenido de las propuestas y se analiza la pertinencia de cada variable como VAESAR (ver figura 2 ). 
VASAR

Variables Ambientales

asociadas a la

Sostenibilidad

Ambiental Regional
VADIT

Variables Ambientales

asociadas a las

Dinámicas Territoriales
VAESAR

Variables Ambientales

Estratégicas para el estudio

de la Capacidad de
Sostenibilidad Ambiental

Regional

Clima
Biodiversidad
Recursos marinos
Agua
Bosques
Suelo/uso del suelo
Vulnerabilidad y
riesgos

$\begin{aligned} & \text { Agua } \\ & \text { Clima } \\ & \text { Suelo/uso del } \\ & \text { suelo } \\ &+\quad \text { Geomorfologia } \\ & \text { Biodiversidad } \\ & \text { Contaminación } \\ & \text { Vulnerabilidad } \\ & \text { y riesgos }\end{aligned}$

Clima

Biodiversidad

Agua

Bosques

Sulo

Vulnerabilidad $y$

riesgos

Contaminación
Trinsito $y$ transporte Población

Energia

Salud

Vivienda

Seguridad ciudadana

Educación

Recreación y cultura

Seguridad social

Agricultura

Empleo

Remuneración al

trabajo

Ingreso y distribución

del ingreso

Finanzas

Ejecución presupuestal

Producción más limpia

Comportamiento fiscal

local

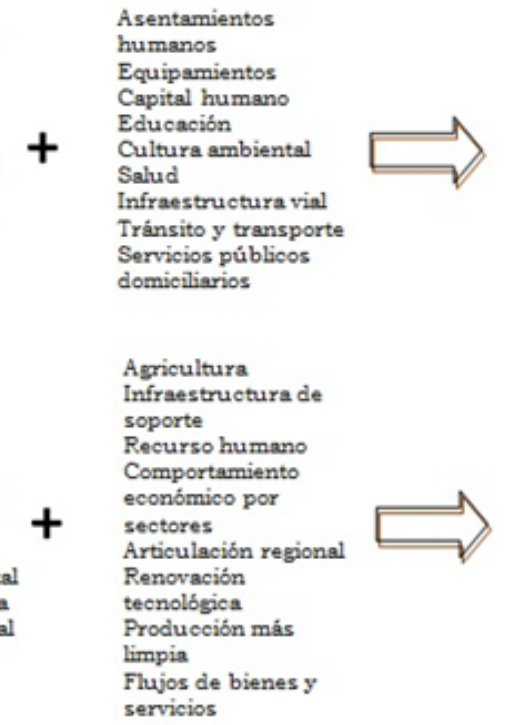

humanos

Equipamientos

apital humano

Cducación

Salud

nfraestructura via

Tránsito y transporte

Servicios públicos

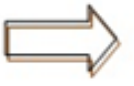

Capital humano

Energía

Equipamientos

Servicios públicos

domiciliarios

Agrieultura

Producción más

limpia

Infraestructura de

soporte

Recurso humano

Flujos de bienes y

servicios

\section{Participación en politica}

Inversión institucion

Inversión ambiental

Capacidad institucional

Participación

ciudadana/comunitaris

Gestión subernamental

Gestión no

Gubernamental

Gubernamental

privado

Gestión ambiental

$\begin{array}{ll} & \text { Organización } y \\ & \text { participación } \\ & \text { comunitaria } \\ +\quad & \text { Gestión } \\ \text { gubernamental } & \text { Coordinación } \\ \text { interinstitucional } \\ \text { Inversión ambiental } \\ \text { Convenios de } \\ \text { cooperación }\end{array}$

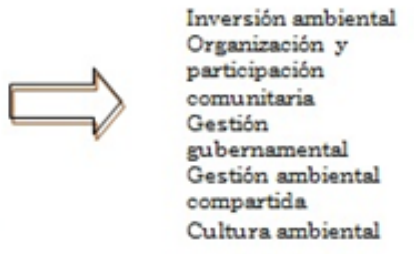

Figura 2. Variables Ambientales Estratégicas VAESAR para el estudio de la capacidad de sostenibilidad ambiental regional.

Las variables ambientales que resultaron estratégicas para el estudio de la capacidad de sostenibilidad ambiental regional en cuanto a la dimensión biofísica son clima, suelo, agua y biodiversidad, las cuales reflejan la capacidad del ecosistema para sostener el socio-sistema, y a su vez, la capacidad de éste para afrontar aquellas restricciones que impone el ecosistema representada en la variable vulnerabilidad y riesgos (figura 2). Resultó también estratégica, la variable bosques, debido a su contribución en la protección del recurso hídrico, como fuente de captura de Carbono, y su impacto frente al calentamiento global, temas que restringen a nivel planetario la sostenibilidad. En este sentido el Banco Interamericano de Desarrollo, plantea "se están también ampliando oportunidades para mitigar las emisiones de $\mathrm{CO} 2$ vinculando el secuestro de carbono y la protección de bosques" (2004: 
185). La variable contaminación, agrupa los diversos tipos de alteración de los componentes del ambiente por la presencia de sustancias o elementos extraños, y permite analizar las causas del deterioro de los componentes ambientales, que contribuyen a disminuirlos, y a poner en riesgo la sostenibilidad ambiental. En conjunto, las variables ambientales de esta dimensión reflejan el potencial con el que cuenta una región como capital natural para sostener las diferentes demandas de su territorio.

Desde la dimensión socio-espacial, la variable tránsito y transporte, resultó estratégica, debido a la influencia negativa que ejerce esta variable sobre la capacidad del capital natural, específicamente el aire, requiriéndose a futuro la minimización del uso de transporte motorizado, o su modificación hacia sistemas menos entrópicos, y al alto consumo de combustibles fósiles. Al respecto

"otro reto y oportunidad importante para la región que recibe cada vez más atención es el mejoramiento de sistemas de transporte público para reducir congestión y costos, además de mejorar acceso y la calidad del medio ambiente en las localidades" ( $p$. 183).

Así mismo, la variable capital humano desde el enfoque de la cantidad y distribución de la población en el territorio, y desde la cualificación del ser humano, debida principalmente al acceso a la educación, al goce de una buena salud base de la productividad económica del individuo y la sociedad, y a las oportunidades de crecimiento personal como factores que contribuyen con su mejoramiento, favorecen el capital social y una mayor disposición a participar responsablemente en los asuntos de gobernabilidad. Otra variable seleccionada, debido a la gran dependencia que genera para el sostenimiento del socio-sistema y teniendo en cuenta su incidencia negativa sobre la sostenibilidad ambiental, fue la variable energía, al considerar el reto que a futuro constituye contar con energías alternativas más amigables con el ambiente, y menos dependientes de recursos o materiales no renovables que disminuyan las presiones sobre el capital natural. "Estas cifras proveen una perspectiva sobre los retos y opciones que la región está enfrentando para lograr los Objetivos de Desarrollo del Milenio relacionados al uso de energía y a las emisiones de dióxido de carbono" (p. 183). Igualmente, dentro de esta dimensión la variable equipamientos contribuye a potenciar las capacidades del capital humano, y afectar el estado del saneamiento básico como determinante también de las condiciones de vida de la población representado en la oferta y calidad de los servicios públicos domiciliarios, con grandes demandas del capital natural e indispensables para la salud física, la seguridad e higiene de la población. Por tal razón: "el logro de los objetivos de agua y saneamiento representa un gran reto para la región, con implicaciones en varios sentidos" (p. 185).

En cuanto a la dimensión económica, las variables ambientales VASAR, a excepción de las variables agricultura y producción más limpia, constituyen elementos para diagnosticar el estado de un sistema, y no se consideran relevantes para estudiar la capacidad de sostenibilidad ambiental, debido a que no demandan capital natural, ni dependen de otras variables más estratégicas, razón por la cual, no se incluyeron como VAESAR. La variable agricultura requiere hacer uso del capital natural asociado a un territorio, el cual se restringe cada vez más, por los manejos inadecuados del suelo y el recurso hídrico principalmente; la variable producción más limpia permite garantizar una producción económica en armonía con el ambiente tras la reducción de los impactos ambientales que generan los diferentes tipos de entropía, lo 
que supone reducir las restricciones ambientales que en materia de comercio exterior se imponen cada vez más. A esta última variable, se integró la variable renovación tecnológica toda vez que una tecnología más eficiente contribuye a disminuir la disipación de entropía, mejora la producción más limpia y optimiza los procesos de la agricultura como fundamentales en la seguridad alimentaria de la población y los ecosistemas.

\begin{abstract}
"Para lograr este objetivo es necesario el trabajo conjunto entre los sectores público y privado, alentando la conservación de los recursos así como el impulso de instrumentos orientados a mejorar la producción bajo criterios de sostenibilidad, como es la certificación ecológica y la comercialización de productos "benignos" para el medio ambiente" (p. 175).
\end{abstract}

Las variables infraestructura de soporte, recurso humano y flujos de bienes y servicios de la propuesta VADIT, responden a la concepción de VAESAR, debido a que reflejan la necesidad de consumo de capital natural, y vinculan el capital socio-espacial principalmente relacionado con el recurso humano para su dinamización.

La dimensión político institucional contiene tres variables ambientales que resultaron comunes a ambas propuestas: inversión ambiental, organización y participación comunitaria y gestión gubernamental, observándose su importancia para mantener la capacidad del gobierno, las instituciones y la comunidad en la vía hacia la sostenibilidad. Por otra parte, se incorporó la variable gestión ambiental compartida, teniendo en cuenta las políticas mundiales frente a la necesidad de articular esfuerzos de todo tipo, y apoyar a los gobiernos en la toma de decisiones efectivas que contribuyan a sostener ambientalmente el territorio como compromiso y responsabilidad de todos, y la variable cultura ambiental de la dimensión socio-espacial de la propuesta VADIT, dada la necesidad urgente de mejorar la formación de ciudadanos comprometidos con la calidad del ambiente y competentes para intervenir en las políticas ambientales de manera consciente, responsable y efectiva. Los programas del Banco Interamericano de Desarrollo procurarán:

"formular y consolidar un marco de gestión ambiental que sea transparente y participativo y que conduzca al establecimiento de prioridades adecuadas en cuanto a la gestión sostenible del capital natural y la calidad del medio ambiente, enfocándose en: (i) el fortalecimiento de las instituciones y la participación de la sociedad civil; (ii) el fortalecimiento de los marcos normativos; y (iii) la formulación de conjuntos eficaces de instrumentos de gestión ambiental” (p. 196).

\section{BIBLIOGRAFÍA}

- Ángel, A. (1996). El reto de la vida. Bogotá: Ecofondo.

- Antequera, J. (2005). El potencial de sostenibilidad de los asentamientos humanos. Desde: http://www.eumed.net/libros/2005/ja-sost/.

- Banco Interamericano de Desarrollo. (2004). Los objetivos de desarrollo del milenio en América Latina y el Caribe: retos, acciones $y$ compromisos. México. 
- Bettini, V. (1998). Elementos de ecología urbana. Madrid: Editorial Trotta.

- Fals, O. (1996). Región e historia. Elementos sobre ordenamiento y equilibrio Regional en Colombia. Bogotá: TM Editores-IEPRI.

- Leal, G. (2006). Introducción al ecourbanismo: el nuevo paradigma. Bogotá: Ecoe Ediciones.

- Morin, E. (2000). El Paradigma perdido. Madrid: Kairós.

- Naredo, J. M. (1997). Sobre la insostenibilidad de las actuales conurbaciones y el modo de paliarla. Obtenido el 5/09/2011. Desde: http://habitat.aq.upm.es/cs/p2/a007.html.

- Parra, F. (1997). Ciudad y entorno natural. Obtenido el 5/09/2011. Desde: http://habitat.aq.upm.es/cs/p3/a015.html.

- Rayén, Q. M. (2001). Indicadores de sostenibilidad ambiental y de desarrollo sostenible: estado del arte y perspectivas. Santiago de Chile: CEPAL.

- Velásquez, L. (2005). Sostenibilidad urbana en América Latina. Cataluña: U. P. Cataluña.

- Yunén, R. (1997). Medio ambiente urbano: marco conceptual. Revista Interamericana de Planificación ¿Quiénes hacen ciudad?, XXIX . pp. 115-116.

- Zuluaga S. G. (2003). La ciudad y el campo. En: Revista Gestión y Ambiente, 6. No. 1

1. Magíster en Planeación Urbano Regional. Profesora Asociada. Universidad Nacional de Colombia, Sede Palmira. mvpinzonb@unal.edu.co. Integrante del grupo de investigación Prospectiva Ambiental. Universidad Nacional de Colombia, Sede Palmira.

2. Magíster en Medio Ambiente y Desarrollo. Docente Ocasional. Universidad Nacional de Colombia, Sede Palmira fisin.2011@gmail.com. Integrante del grupo de investigación Prospectiva Ambiental. Universidad Nacional de Colombia, Sede Palmira. 